\title{
Embedded Nanoparticle Analysis using Atom Probe Tomography and High-Resolution Electron Microscopy
}

\author{
Satyanarayana V. N. T. Kuchibhatla ${ }^{1}$, V. Shutthanandan ${ }^{1}$, B.W. Arey ${ }^{1}$, L. Kovarik ${ }^{1}$, C.M. Wang ${ }^{1}$, \\ T. J. Prosa ${ }^{2}$, R. M. Ulfig ${ }^{2}$, S. Thevuthasan ${ }^{1}$, B. P. Gorman ${ }^{3}$ \\ ${ }^{1}$ EMSL, Pacific Northwest National Laboratory, Richland, WA 99354 satya@pnl.gov \\ ${ }^{2}$ Cameca Instruments Inc., Madison, WI 53711 \\ ${ }^{3}$ Colorado School of Mines, Golden, CO 80401
}

One of the major challenges before the materials scientists today is the ability to "determine the positions (in 3D) and chemical identity of individual atoms in any materials system". It is envisioned that the combination of atom probe tomography, APT, and the advances in high-resolution electron microscopy paves the way towards this goal. APT is primarily a combination of point-projection microscopy and time-of-flight mass spectrometry [1]. The three dimensional atomic structure of the sample tips can be obtained through a reconstruction of the experimental data generated by a progressive layer-by-layer evaporation of the atoms from the needle-shaped tip and accounting for the time-of-flight of the ions. Recently, the development of laser-assisted evaporation of materials has unveiled the great potential of APT to analyze the insulating materials. APT is shown to provide chemical identity of the samples with atomic-scale resolution with a field-of-view better than $100 \mathrm{~nm} \times 100 \mathrm{~nm} \times 100 \mathrm{~nm}$. Despite these interesting features, there exist various challenges in terms of data analysis and the assumptions made while carrying out the reconstruction process. It is hence possible to compliment the APT data with high-resolution electron microscopy and thereby obtain a more realistic picture of the material under consideration.

Here, we explore the possibility of the intelligent marriage between APT and TEM/STEM to obtain the three-dimensional chemical imaging of Au-nanoparticles embedded in a dielectric (such as $\mathrm{MgO}$ ) matrix. Ion beam implantation of $\mathrm{Au}+(2 \mathrm{MeV})$ in oxides and post-implantation annealing treatments have been shown to result in in situ nanoparticle formation. Previously, it was indicated by the researchers that the embedded structures are of pure-Au, based on the electron microscopy and optical absorption analyses [2]. However, the recent atom probe tomography (APT) analysis of the Au-implanted MgO samples questions these results and the first set of conclusions indicate that the nanoclusters formed within the $\mathrm{MgO}$ matrix may consist of a mixture of $\mathrm{Au}, \mathrm{Mg}$ and $\mathrm{O}$ as opposed to $100 \% \mathrm{Au}$ as reported and further validation is in progress.

Detailed APT analysis is underway at the EMSL, Environmental Molecular Sciences Laboratory, to gain the clear understanding about the chemical composition of these nanoclusters as they may have implications in sensors and catalysis related research. The samples were analyzed under different laser pulse energies $(0.3 \mathrm{~nJ}-0.8 \mathrm{~nJ}, 542 \mathrm{~nm})$, which indicate that the higher laser energies may lead to various artifacts including non-stoichiometric $\mathrm{MgO}$ mass fractions. Further analysis was carried out on the sample run at $0.3 \mathrm{~nJ}$ pulse energy and the iso-concentration surface analysis revealed that the Au-particle size is similar to that observed from TEM and STEM. While the size of the clusters is shown to increase with the increasing depth of implantation, the 1D-compositional analysis of the clusters indicates that the largest cluster may contain a maximum of up to $50-60 \%$ Au with the others having relatively lower concentration of $\mathrm{Au}$. The interface between the nanoparticle and the $\mathrm{MgO}$ matrix is often rich with $\mathrm{Mg}$ and $\mathrm{O}$ and the Au-particles were observed to contain stoichiometric $\mathrm{Mg}$ and $\mathrm{O}$. No significant clustering was observed in the case of $\mathrm{MgO}$. Some of the interesting observations during this analysis also include the variations in the mass resolution as a function of $\mathrm{Au}$ concentration. 
In order to validate the data further, atom probe tips were prepared on half-cut TEM grids utilizing the focus ion beam technique and [3] analyzed in both high-resolution electron microscope and APT. High-resolution STEM analysis revealed the coherent nature of the Au-nanoparticles in the MgO matrix. The results will be discussed in detail while drawing the attention towards the "strengths" of complimentary imaging.

\section{References}

[1] M.K. Miller et al., Atom Probe Field Ion Microscopy, Oxford University Press, Oxford, 1996.

[2] Wang et al., Applied Physics Letters 87, 153104, 2005

[3] B. P. Gorman et al., Microscopy Today, June/July 2008
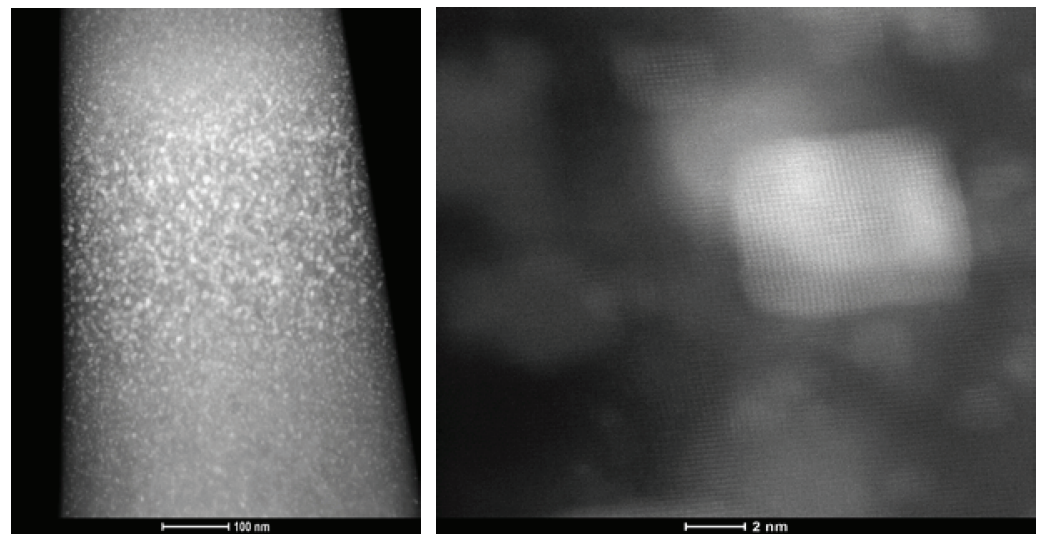

Typical STEM - HAADF images obtained from aberration corrected TITAN instrument at EMSL.

Left: Image of an APT tip

Right: High-magnification image showing the coherent Au-particle in $\mathrm{MgO}$
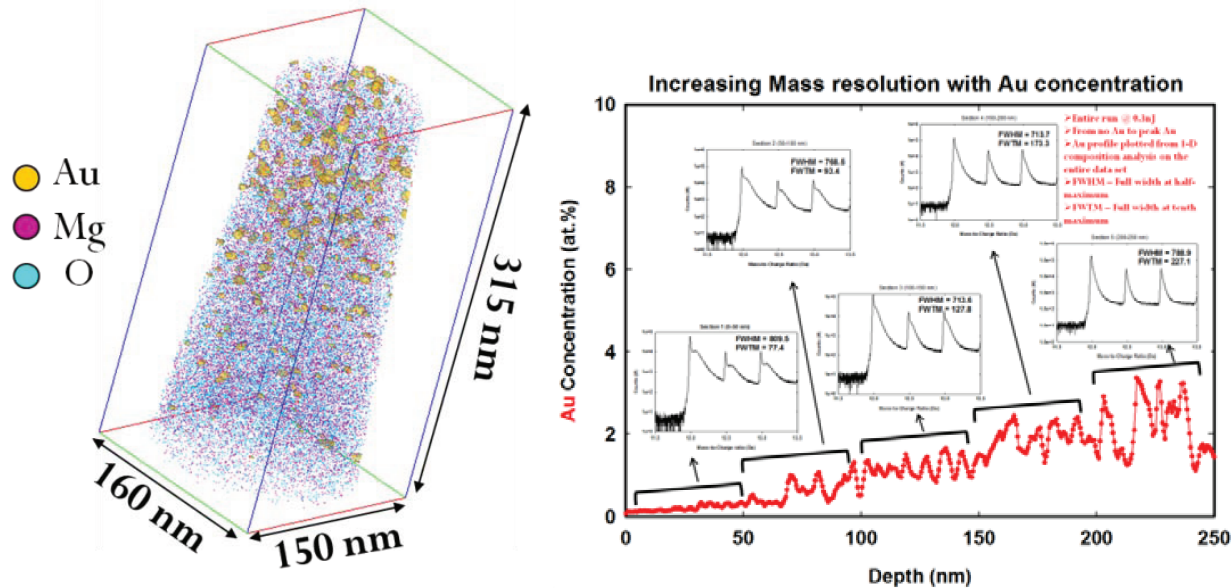

Left): 3-D reconstructed image of APT sample showing the Auiso-concentration surfaces

Right): View graph indicating the variation in the mass resolution with increasing Auconcentration 